\title{
Comparison between a FEL amplifier and oscillator
}

\author{
P. Zambon ${ }^{\mathrm{a}, *}$, W.J. Witteman ${ }^{\mathrm{a}}$, P.J.M. van der Slot ${ }^{\mathrm{b}}$ \\ ${ }^{a}$ Unversity of Twente, Department of Appled Physics, P O. Box 217, 7500 AE Enschede, The Netherlands \\ ${ }^{b}$ Nederlands Centrum voor Laser Research B.V., P.O. Box 2662, 7500 CR Enschede, The Netherlands
}

Previous experiments with the Raman FEL, situated at the Twente University, showed that the output was influenced by the rather strong increase of the current density with time. The field emission diode has been modified to produce a more constant current pulse to simplify the analysis of the measurements. This resulted in a lower current density of the electron beam. With this new diode two set-ups are studied. In the first set-up the laser is still configured as an amplifier whereas in the second set-up the laser configuration is changed into an oscillator using a Bragg reflector with a space-variable corrugation height. For both set-ups we measured the frequency spectrum for specific values of undulator and guide magnetic fields. The relative performance of the amplifier and the oscillator configuration will be presented.

\section{Introduction}

In previous experiments with the Twente Raman FEL the physics of the laser configured as an amplifier were investigated [1]. Briefly, the main parameters of the Twente Raman FEL are an accelerating voltage of $500 \mathrm{kV}$ for a duration of $100 \mathrm{~ns}$ and a maximum peak current of the electron beam of $\approx 900 \mathrm{~A}$. The helical undulator has a period of $3 \mathrm{~cm}$ and a tapered entrance of 6 periods. The waveguide has an inner radius of 8 $\mathrm{mm}$.

To be able to compare the performance in an amplifier configuration with that of an oscillator it is necessary to reduce the maximum current of the electron beam. Moreover, the value of the impedance was not constant during a shot, resulting in a strong increase (typically from $600 \mathrm{~A}$ to $900 \mathrm{~A}$ ) of the current during the shot which could result in a frequency shift during the pulse [1]. By increasing the anode-cathode distance in the field emission diode (FED) to $1.5 \mathrm{~cm}$ it is found that the maximum current decreased to typically $200 \mathrm{~A}$ whereas the time dependence has been reduced.

A Bragg reflector [2] (downstream mirror) and a metal plate with a hole (upstream mirror) are used to create a resonator with an overall reflectivity of 0.3 at the design frequency of $30 \mathrm{GHz}$. The first experiments, reported here, were aimed at comparing the spectra obtained with and without Bragg reflector for different

\footnotetext{
* Corresponding author
}

values of the axial magnetic field at one setting for the undulator magnetic field. Using the intersection of the dispersion relation for the $\mathrm{TE}_{11}$ waveguide mode and the slow space-charge wave, one finds that the FEL interaction occurs at $30 \mathrm{GHz}$ for an axial guide field of $B_{z}=1.0 \mathrm{~T}$ and an undulator field of $B_{\mathrm{u}}=0.12 \mathrm{~T}$ with a current $I=200$ A.

In next section the experimental set-up is described, in section 3 the preliminary results are presented together with a discussion and a summary with concluding remarks is given in section 4 .

\section{Experimental set-up}

The amplifier configuration is the same as in previous experiments $[1,3]$. For the oscillator a Bragg reflector was mounted just before the outcouple horn, thereby increasing the cavity length with $0.27 \mathrm{~m}$. The radiation emitted by the laser is received by two sectoral horns placed symmetrically around the axis of the laser at a distance of $0.93 \mathrm{~m}$ from the outcouple horn. The radiation is guided to a shielded room using two standard rectangular $\mathrm{K}_{\mathrm{a}}$-band waveguides. Two threeway power splitter are used to feed six cut-off waveguide filters [3], F8, $\lambda_{\mathrm{c}}=8 \mathrm{~mm}$, to $\mathrm{F} 13, \lambda_{\mathrm{c}}=13 \mathrm{~mm}$ with $1 \mathrm{~mm}$ increment. The radiation which passes through a filter is detected by a HP R422C crystal detector. Two filter signals were delayed by 150 and 300 ns respectively and combined with a third filter signal. In this way the six filter signals could be mea- 
sured using only 2 channels on a fast digitising oscilloscope. The signal analysis was done in a similar way as described in refs. [1,3].

Before describing the preliminary results it must be emphasised that a small reflection $(\leq-20 \mathrm{~dB})$ is still present in the amplifier configuration because of the presence of a vacuum window in the outcouple horn [1]. Furthermore only frequencies above the cut-off frequency of the $\mathrm{K}_{\mathrm{a}}$-band waveguide $\left(f_{\mathrm{c}}=21.08 \mathrm{GHz}\right)$ can be measured with the current set-up. Therefore only part of the spectra can be measured and the attention is focused on the upper intersection of the waveguide modes with the slow plasma wave.

\section{Amplifier and oscillator spectra comparison}

First the total energy received by the sectoral horns has been measured. No correction has been made for the free-space propagation and the attenuation of the transport waveguide to the shielded room. The results are presented in Fig. 1 for different values of the axial

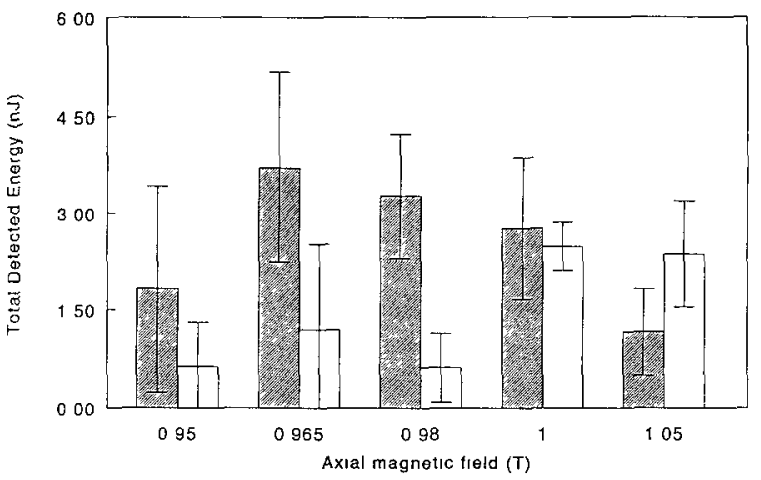

Fig. 1. Total energy above $22 \mathrm{GHz}$ for the amplifier (shaded bars) and oscillator (open bars) configuratıon. The measurements were not corrected for the coupling attenuation due to free space propagation between the outcouple horn and the sectoral horns and the attenuation of the transport waveguide.

guide field at an undulator field of $B_{\mathrm{u}}=0.12 \mathrm{~T}$. The error bars represent a shot to shot spread. It should be remarked that for $B_{z}=1.05 \mathrm{~T}$ the peak value of the
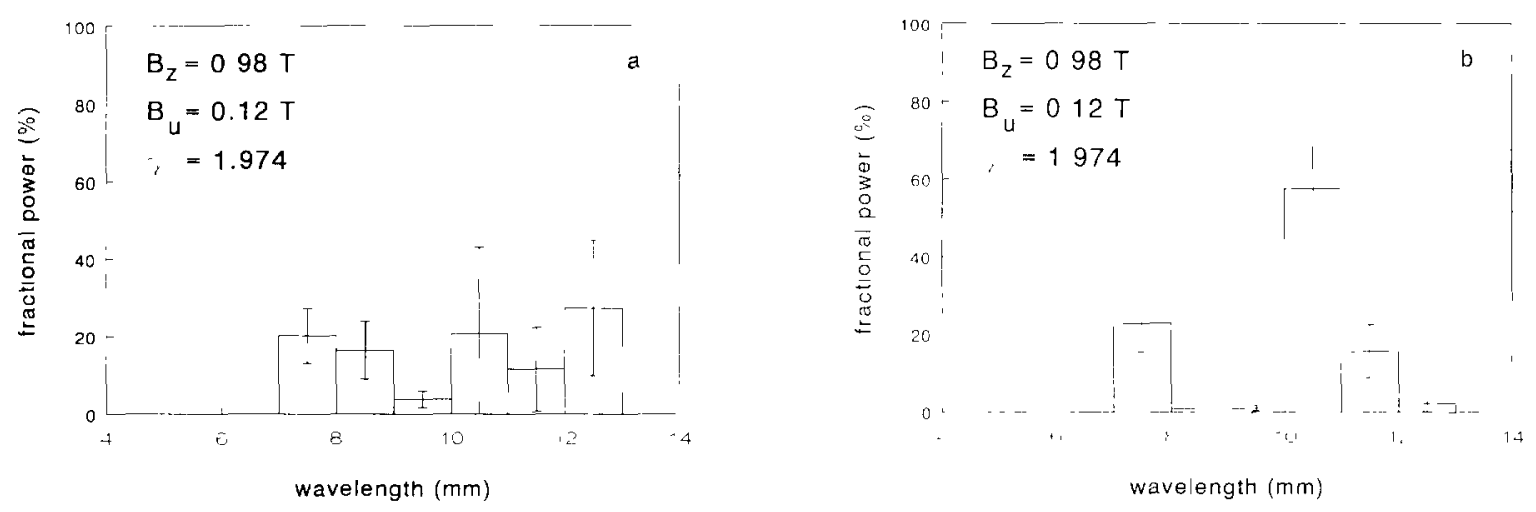

Fig. 2. Measured spectrum with $B_{z}=0.98 \mathrm{~T}$ and $B_{\mathrm{u}}=0.12 \mathrm{~T}$ for the amplifier configuration (a) and for the oscillator configuration (b).
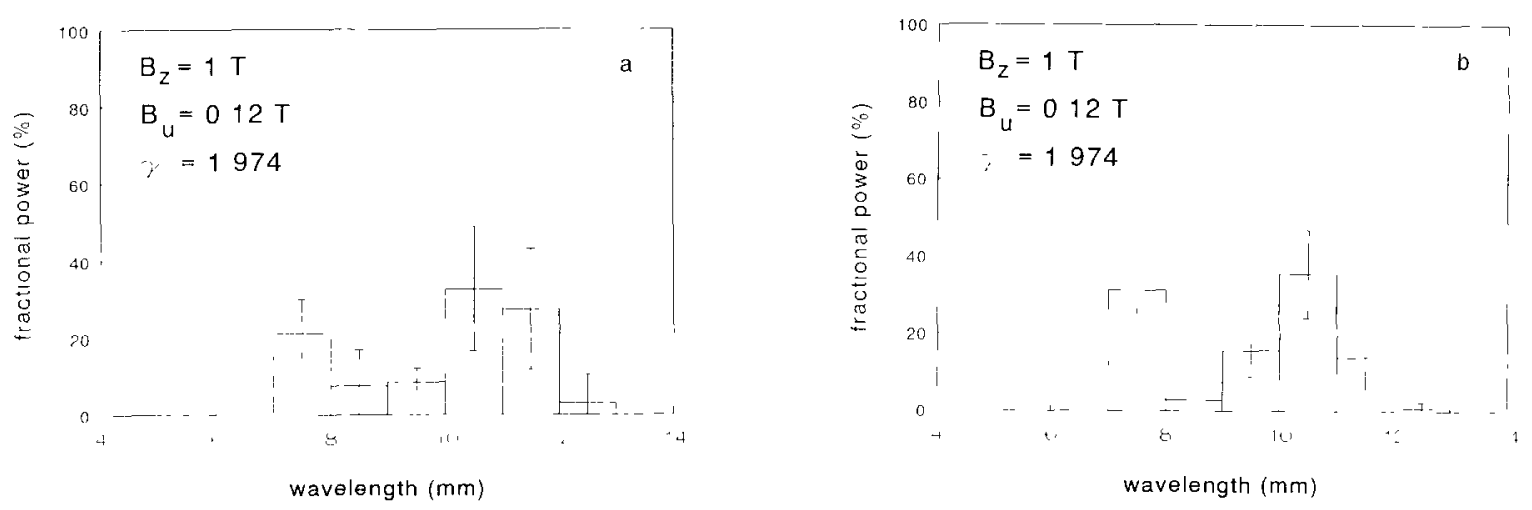

Fig. 3. As Fig. 2 but with $B_{z}=1.00 \mathrm{~T}$ and $B_{\mathrm{u}}=0.12 \mathrm{~T}$ 

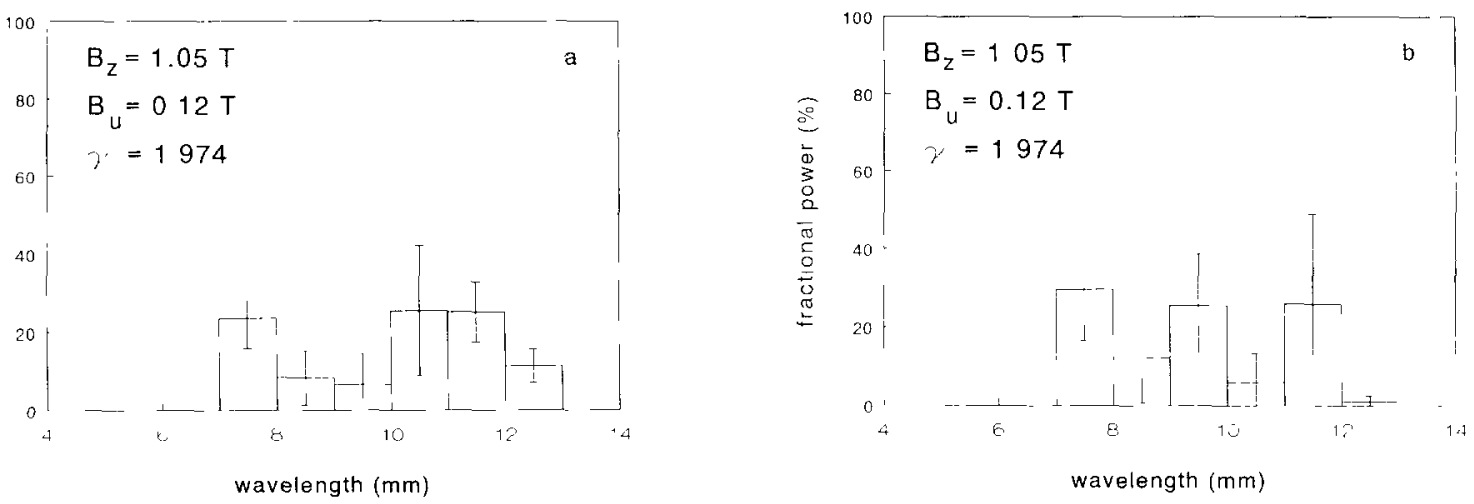

Fig. 4. As Fig. 2 but with $B_{z}=1.05 \mathrm{~T}$ and $B_{\mathrm{u}}=0.12 \mathrm{~T}$.

current was on average some 1.4 times larger when the Bragg reflector was installed compared to the measurements done without the Bragg reflector whereas for $B_{2}=0.95 \mathrm{~T}$ this factor was 1.3 . The peak current was approximately the same for the rest of the settings investigated. It is remarkable that the oscillator configuration gave systematically lower total energy emitted above $21.08 \mathrm{GHz}$. At this moment no conclusion can be drawn from these observations since not enough information is available. First one needs to know if the laser has saturated in the amplifier and in the oscillator configuration ${ }^{\# 1}$. Second the fixed position of the receiving horns results in different coupling (i.e. ratio of received energy compared to total generated radiation energy in the laser) for different modes. However this information is useful in combination with the spectra measured using the two configurations.

The spectra have been measured for the calculated setting $\left(B_{\mathrm{u}}=0.12 \mathrm{~T}\right.$ and $B_{z}=1.0 \mathrm{~T}$, resulting in FEL

\#1 In future experiments it is planned to vary the interaction length in order to investigate this effect.

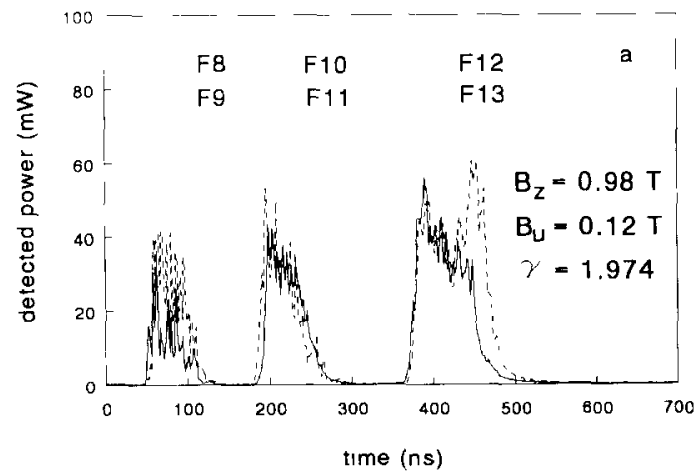

interaction with $\mathrm{TE}_{11}$ mode at $30 \mathrm{GHz}$ ) and neighbouring settings $B_{z}=0.95,0.97,0.98 \mathrm{~T}$ and $1.05 \mathrm{~T}$. The spectra for $B_{z}=0.98,1.00$ and 1.05 are shown in Figs. 2,3 and 4 respectively. Note the bar at $8 \mathrm{~mm}$ represents the fractional energy emitted at wavelengths $\leq 8$ $\mathrm{mm}$. All measured spectra (i.e. also the ones not shown here) indicate a suppression of the long wavelength $(\lambda \geq 12 \mathrm{~mm})$ components when the Bragg reflector is installed. This is surprising since calculations show that the Bragg reflector should be transparent (i.e. the transmission coefficient $T=1$ ) for wavelengths above $11 \mathrm{~mm}$. One may therefore conclude that there is a coupling between the radiation emitted at a longer wavelength with that emitted at a shorter wavelength where the Bragg reflector will influence the FEL interaction.

For $B_{z}=0.98 \mathrm{~T}$ and $1.00 \mathrm{~T}$ it is found that the Bragg reflector reduces the spectral width of the emitted radiation. This is expected if the peak of the gain curve coincides with the peak of the reflection band of the oscillator. The narrowing of the spectrum is more evident for the $B_{z}=0.98 \mathrm{~T}$ setting which may indicate

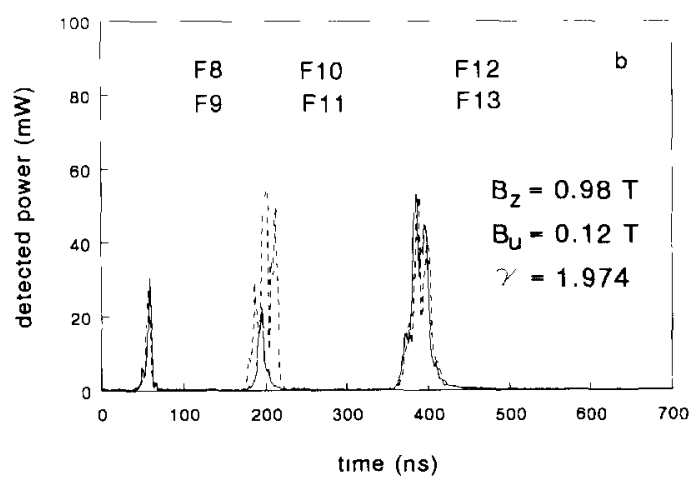

Fig. 5. Detected power with $B_{z}=0.98 \mathrm{~T}$ and $B_{\mathrm{u}}=0.12 \mathrm{~T}$ for the amplifier (a) and oscillator (b) configuration. Signals from the even number filters are represented with a solid line, the dashed line refers to the odd ones. 
that the calculated value of the axial guide field for which resonance at $30 \mathrm{GHz}$ is found is slightly too large. Although most of the power is emitted between 10 and $11 \mathrm{~mm}$ for the $0.98 \mathrm{~T}$ setting (Fig. 2b), the total radiation energy at this wavelength interval is higher for the $1.00 \mathrm{~T}$ setting (cf. Figs. 1, 2 and 3). The suppression of radiation between 8 and $9 \mathrm{~mm}$ may be explained by mode conversion [2]. Whenever the sum of the axial wavenumbers of two modes (with the same azimuthal dependence) equals the Bragg wavenumber of the reflector the incident mode is reflected in the other mode. For this particular Bragg reflector we have $\mathrm{TE}_{11}$ (incident) $\rightarrow \mathrm{TE}_{11}$ (reflected) at $30 \mathrm{GHz}(10 \mathrm{~mm})$ and $\mathrm{TE}_{11} \rightarrow \mathrm{TM}_{11}$ at $33.4 \mathrm{GHz}(8.9 \mathrm{~mm})$. It can therefore be expected that around $8.9 \mathrm{~mm}$ an incident $\mathrm{TE}_{11}$ mode is reflected into a $\mathrm{TM}_{11}$ mode which may not couple to the electron beam.

The reduction in total emitted energy for the $0.98 \mathrm{~T}$ setting of the guide field when a Bragg reflector is installed is related to the time structure of the emitted radiation pulse. In Fig. 5 typical filter signals as measured with the crystal detectors are plotted for the configuration without (Fig. 5a) and with (Fig. 5b) Bragg reflector. The width of the radiation pulse equals the width of the electron pulse when the Bragg reflector is not installed. The pulse width reduces to about $30 \mathrm{~ns}$ for the oscillator configuration. This value corresponds to approximately two round trips in the cavity. The peak power emitted in both configurations is approximately equal but the radiation pulse is about a factor of 3 shorter and, consequently, contains less energy in the oscillator configuration.

For the amplifier it is evident from Fig. 5a that the radiation pulse contains two contributions. When the signals of the filters with the lower cut-off wavelength (F8, $\lambda_{\mathrm{c}}=8 \mathrm{~mm}$ to F12, $\lambda_{\mathrm{c}}=12 \mathrm{~mm}$ ) begin to fall off the signal from filter $\mathrm{F} 13\left(\lambda_{\mathrm{c}}=13 \mathrm{~mm}\right)$ still increases. The difference between the F12 and F13 signals is the power emitted between 12 and $13 \mathrm{~mm}$. It is clear that the radiation with a wavelength between 12 and $13 \mathrm{~mm}$ is emitted at the end of the electron pulse whereas the majority of the energy at shorter wavelength $(\approx 10$ $\mathrm{mm}$ ) is emitted in the first part of the radiation pulse. The increase of the current from 160 to $200 \mathrm{~A}$ during the pulse changes the plasma frequency and thereby shifts the resonance frequency to a lower value by 0.5 $\mathrm{GHz}$. This change is not enough to explain the shift observed in the experiment. From Fig. $5 b$ it can be seen that the radiation is only emitted during the first part of the radiation pulse for the oscillator configuration. It thus seems that the Bragg reflector suppresses the emission at longer wavelength at the end of the electron pulse for reasons not yet understood.

A similar effect can be seen at the $1.0 \mathrm{~T}$ setting of the guide field (see Figs. 6a-6c). The laser configured as amplifier seemed however to operate in two modes
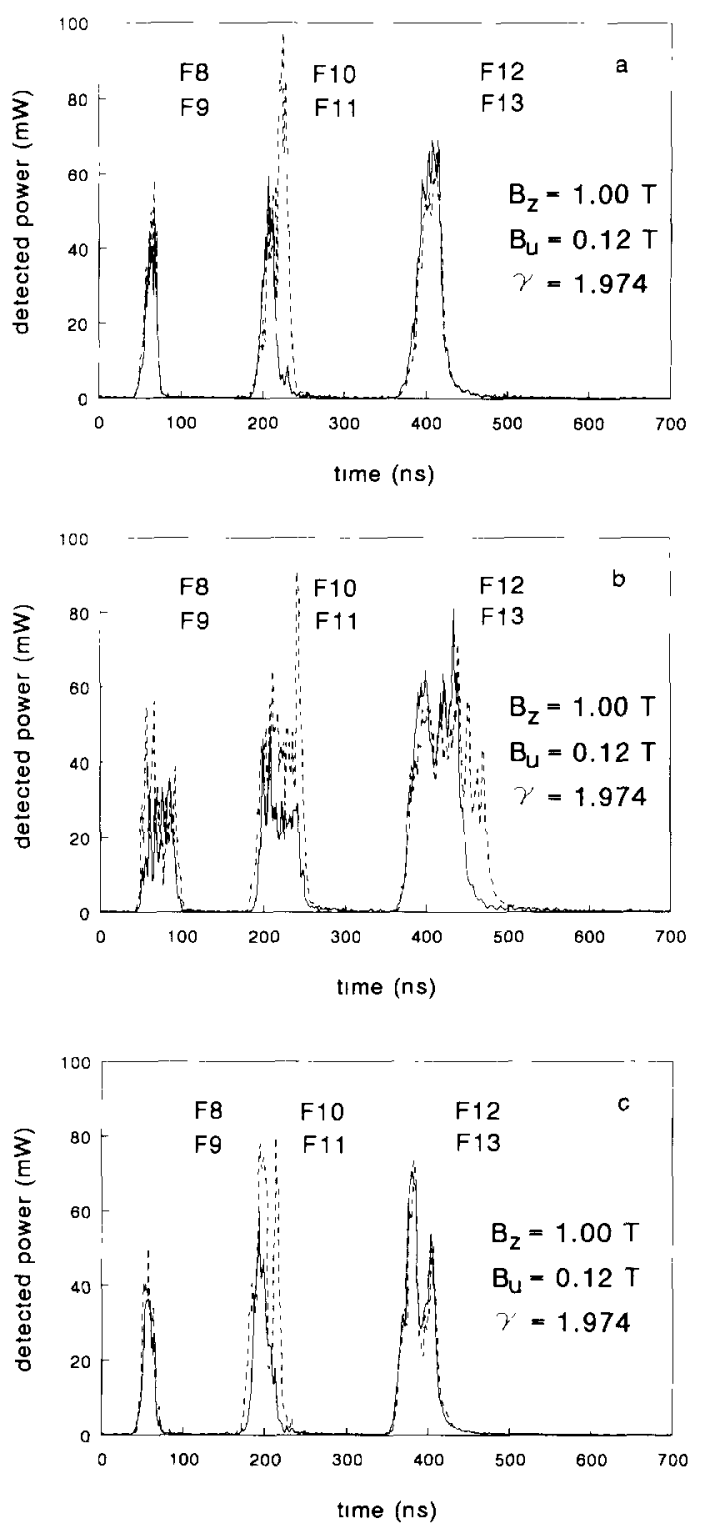

Fig. 6. As Fig. 5 but with $B_{z}=1.00 \mathrm{~T}$ and $B_{4}=0.12 \mathrm{~T}$ for the amplifier (a, b) and oscillator (c) configuration.

as about three quarter of the shots behaved like shown in Fig. 6a whereas the rest behaved as shown in Fig. $6 \mathrm{~b}$. The oscillator configuration was more stable as all shots showed a time behaviour as shown in Fig. 6c.

\section{Conclusions}

The peak current used in the Twente Raman FEL has been reduced to about $200 \mathrm{~A}$. The laser has been configured as an amplifier and as an oscillator and 
some preliminary results have been presented. It is found that in general the total energy emitted above 22 $\mathrm{GHz}$ is lower in the oscillator set-up compared to the amplifier set-up. This is partially related to the shorter duration of the radiation pulse found for the oscillator for the parameters investigated. For the amplifier it is found that, at the $0.98 \mathrm{~T}$ setting of the guide field, the radiation pulse contains two contributions, one at long wavelength $(\approx 12.5 \mathrm{~mm})$ which appears at the end of the electron pulse and one at shorter wavelength $(\approx$ $10.5 \mathrm{~mm}$ ) emitted during the first part of the electron pulse. With the oscillator configuration it is found that the contribution at longer wavelength is no longer present. It is also found that the spectral width of the radiation has been reduced in the oscillator configuration for the $0.98 \mathrm{~T}$ and $1.0 \mathrm{~T}$ setting of the guide field.

\section{Acknowledgements}

The authors would like to thank P.J.S. Teunisse from Hollandse Signaalapparaten B.V., The Netherlands and A.F.M. Bouman for their assistance with the rf diagnostics.

\section{References}

[1] P.J.M. van der Slot and W.J. Witteman, Nucl. Instr. and Meth. A 331 (1993) 140.

[2] P. Zambon and P.J.M van der Slot, these Proceedings (15th Int. Free Electron Laser Conf., The Hague, The Netherlands, 1993) Nucl. Instr. and Meth. A 341 (1994) 484.

[3] P.J.M van der Slot, Ph.D. Thesis, University of Twente, The Netherlands (1992). 